\title{
SERIES ASSOCIATED WITH CERTAIN IRREGULAR THIRD-ORDER BOUNDARY VALUE PROBLEMS*
}

BY

\author{
LEWIS E. WARD
}

1. Introduction. In a previous paper $\dagger$ the writer discussed the characteristic functions of the differential system

$$
u^{\prime \prime \prime}+\rho^{3} u=0, u(\pi)=u\left(-\omega_{2} \pi\right)=u\left(-\omega_{3} \pi\right)=0,
$$

where $\omega_{2}=e^{\pi i / 3}$ and $\omega_{3}=e^{-\pi i / 3}$, and showed that these characteristic functions fall naturally into three classes, denoted by $u_{1 k}(x), u_{2 k}(x)$, and $u_{3 k}(x)$. It was proved that if $f(x)$ is a function which can be written in the form $\phi_{1}\left(x^{3}\right)+x \phi_{2}\left(x^{3}\right)+x^{2} \phi_{3}\left(x^{3}\right)$, where each $\phi$ represents a convergent power series in $x^{3}$ and is such that $\phi_{1}, \phi_{2}$, and $\phi_{3}$ have continuous second, third, and fourth derivatives respectively in the interval $0 \leqq x \leqq \pi, \ddagger$ then $f(x)$ can be expanded in a series of these characteristic functions

$$
\sum_{k=1}^{\infty}\left[a_{1 k} u_{1 k}(x)+a_{2 k} u_{2 k}(x)+a_{3 k} u_{3 k}(x)\right],
$$

the series converging uniformly to $f(x)$ in the interior and on the boundary of some equilateral triangle $T_{1}$ centered at $x=0$, having one vertex on the segment from 0 to $\pi$, and lying inside the smallest of the circles of convergence of the power series for the $\phi$ 's.

The purposes of this paper are to consider the uniqueness of this expansion, to extend the region of uniform convergence, to consider the differentiation and the integration of the series, to prove certain relations involving the coefficients, and to show how the coefficients may be obtained by rendering stationary certain integrals.

2. Uniqueness of the expansion. The coefficients in the expansion (1),

* Various parts of this paper were presented to the Society on April 7, 1928, March 29, 1929, and August 29, 1929; the paper was received by the editors October 23, 1929. 745.

$\dagger$ Some third-order irregular boundary value problems, these Transactions, vol. 29 (1927), pp. 716-

$\ddagger$ Ward, loc. cit., Theorem 1, p. 742. The continuity of the second derivatives only of $\phi_{2}$ and $\phi_{3}$ is sufficient in the present case. The continuity of the higher derivatives was demanded in the theorem referred to because that theorem is based on Theorem 2, p. 730 of the same paper, in the proof of which this continuity was ustful in connection with the formal relations between the $\phi$ 's of the latter theorem. There need be no formal relations between the $\phi$ 's of Theorem 1, p. 742, nor between the $\phi$ 's of the present paper. 
called the formal coefficients, arè obtained from certain orthogonality relations, as is customary in expansion problems associated with differential systems. Explicit formulas for them are

$$
a_{i k}=\frac{1}{\pi \delta_{i-1}\left(\rho_{i k} \pi\right)} \int_{0}^{\pi} x^{i-1} \phi_{i}\left(x^{3}\right) \delta_{3}\left[\rho_{i k}(\pi-x)\right] d x \quad(i=1,2,3)
$$

where

$$
\delta_{j}(t)=e^{\omega_{1} t}+\left(-\omega_{3}\right)^{j-1} e^{\omega_{2} t}+\left(-\omega_{2}\right)^{i-1} e^{\omega_{3} t}, \omega_{1}=-1 \text {, and } \delta_{j}(t)=\delta_{j+3}(t) . *
$$

The $u_{i k}(x)$ of the introduction are given by $u_{i k}(x)=\delta_{i}\left(\rho_{i k} x\right)$.

It was shown in the paper referred to that each of the expansions

$$
\sum_{k=1}^{\infty} a_{i k} u_{i k}(x)=x^{i-1} \phi_{i}\left(x^{3}\right) \quad(i=1,2,3)
$$

is valid in $T_{1}$. Hence, taking three special cases,

$$
1=\sum_{k=1}^{\infty} b_{1 k} u_{1 k}(x), \quad x=\sum_{k=1}^{\infty} b_{2 k} u_{2 k}(x), \quad x^{2}=\sum_{k=1}^{\infty} b_{3 k} u_{3 k}(x)
$$

It is easily shown by straightforward computation that no one of the $b$ 's is zero, and that the triangle of uniform convergence $T_{2}$ of each of these series can have one vertex anywhere we choose to place it on the interval $0<x<\pi . \dagger$

Since the terms of each of series (3) are analytic functions, the series may be differentiated term by term as many times as we like. Differentiating three times and making use of the fact that $u_{i k}^{\prime \prime \prime}=-\rho_{i k}^{3} u_{i k}(x)$, we obtain

$$
0=\sum_{k=1}^{\infty} b_{i k} \rho_{i k} u_{i k}(x) \quad(i=1,2,3) .
$$

Each of these series converges uniformly in and on $T_{2}$. Adding series (5) for $i=1,2$, and 3 to series (1) produces a series

$$
\sum_{k=1}^{\infty}\left[c_{1 k} u_{1 k}(x)+c_{2 k} u_{2 k}(x)+c_{3 k} u_{3 k}(x)\right]
$$

which converges uniformly to $f(x)$ in and on the smaller of $T_{1}$ and $T_{2}$, and has coefficients different from the formal coefficients.

Instead of expanding $1, x$, and $x^{2}$, as in (4), we may expand $p_{1}\left(x^{3}\right)$, $x p_{2}\left(x^{3}\right)$, and $x^{2} p_{3}\left(x^{3}\right)$, where the $p$ 's are polynomials in $x^{3}$, and, by differ-

* See p. 720 of my former paper, already referred to, for other formulas for the $\delta$ functions, and p. 741 for the $\rho_{i k}$.

$\dagger$ We shall use the notation $T_{i}$ to represent equilateral triangles centered at $x=0$ and having one vertex on the positive axis of reals. 
entiating these the requisite number of times, obtain series converging uniformly to zero in and on $T_{2}$. Adding these series to (1) produces still other series of the type (1) converging uniformly to $f(x)$ in and on the smaller of $T_{1}$ and $T_{2}$ but having coefficients different from the formal coefficients.

It appears from the above that the expansion (1) is by no means unique, there being infinitely many expansions of this type, all converging uniformly to $f(x)$ in a common triangle, which, in case the singularities of $f(x)$ are all more distant from the origin than $\pi$, has one vertex as close to $\pi$ as we care to place it. It is of interest to note the difference between this situation and that which obtains with power series, in which the coefficients are unique however small the circle of convergence, and with Fourier series, in which the coefficients are unique if the series is made to converge to the given function over the interval $(0,2 \pi)$ except possibly at a finite number of points. Of course, if the expansion (1) converges uniformly to $f(x)$ in the interval $0 \leqq x \leqq \pi$, the coefficients are unique.

3. Extension of triangle of uniform convergence. Let us suppose that one at least of the power series for the $\phi$ 's fails to converge for $x=a$ and that all three series converge for $|x|<|a|$. Laying aside for the moment the cases in which $|a| \geqq \pi$, let us suppose $|a|<\pi$. Construct the circle $|x|=|a|$, and let $x=b$ be the point where this circle cuts the axis of reals, $0<b<\pi$. Let $T_{b}$ be the equilateral triangle with one vertex at $x=b$. Then it is known that series (3) converge uniformly to the respective functions standing on the right-hand sides of equations (3) in and on the boundary of every closed region interior to $T_{b}{ }^{*}$ Let $T_{a}$ be the equilateral triangle with one side through $x=a$, and $T_{x}$ the equilateral triangle with one vertex at $\pi$.

THEOREM I. The formal series $\sum_{k=1}^{\infty} a_{i k} u_{i k}(x)$ converges uniformly to $x^{i-1} \phi_{i}\left(x^{3}\right)$ in and on the boundary of every closed region interior to the smaller of $T_{a}$ and $T_{\pi}$.

Let $z$ be that vertex of $T_{a}$ which is on the positive axis of reals if that vertex lies to the left of $\pi$, otherwise let $z=\pi$. Choose $y$ on this axis, $0<y<z$. We shall establish uniform convergence in the interval $0 \leqq x \leqq \alpha<y$, from which the theorem follows immediately. $\dagger$

We examine the magnitudes of the coefficients $a_{i k}$. We have

$$
\int_{0}^{\pi} x^{i-1} \phi_{i}\left(x^{3}\right) \delta_{3}\left[\rho_{i k}(\pi-x)\right] d x=\left[\int_{0}^{y}+\int_{y}^{\pi}\right] x^{i-1} \phi_{i}\left(x^{3}\right) \delta_{3}\left[\rho_{i k}(\pi-x)\right] d x .
$$

But

* Ward, loc. cit., Theorem I, p. 742.

$\dagger$ Ward, loc. cit., Theorem II, p. 742. 
1930]

$$
\left|\int_{y}^{\pi} x^{i-1} \phi_{i}\left(x^{3}\right) \delta_{3}\left[\rho_{i k}(\pi-x)\right] d x\right|<3 M_{1}\left|e^{\omega_{3} \rho_{i k}(\pi-y)}\right|
$$

where $M_{1}=\max \left|x^{i-1} \phi_{i}\left(x^{3}\right)\right|$ on the interval $0 \leqq x \leqq \pi$. Also

$$
\begin{aligned}
& \int_{0}^{y} x^{i-1} \phi_{i}\left(x^{3}\right) \delta_{3}\left[\rho_{i k}(\pi-x)\right] d x \\
& =e^{\omega_{1} \rho_{i k} \pi} \int_{0}^{y} x^{i-1} \phi_{i}\left(x^{3}\right) e^{-\omega_{1} \rho_{i k} x} d x-\omega_{2} e^{\omega_{2} \rho_{i k} \pi} \int_{0}^{y} x^{i-1} \phi_{i}\left(x^{3}\right) e^{-\omega_{2} \rho_{i k} x} d x \\
& -\omega_{3} e^{\omega_{3} \rho_{i k} \pi} \int_{0}^{y} x^{i-1} \phi_{i}\left(x^{3}\right) e^{-\omega_{3} \rho_{i k} x} d x \\
& =e^{\omega_{1} \rho_{i k} \pi} \int_{0}^{y} x^{i-1} \phi_{i}\left(x^{3}\right) e^{\rho_{i k x}} d x+\left(-\omega_{3}\right)^{i+2} e^{\omega_{2} \rho_{i k} \pi} \int_{0}^{-\omega_{2} y} x^{i-1} \phi_{i}\left(x^{3}\right) e^{\rho_{i k} x} d x \\
& +\left(-\omega_{2}\right)^{i+2} e^{\omega_{3} \rho_{i k} \pi} \int_{0}^{-\omega_{3} y} x^{i-1} \phi_{i}\left(x^{3}\right) e^{\rho_{i k} x} d x \\
& =\delta_{i}\left(\rho_{i k} \pi\right) \int_{0}^{-y / 2} x^{i-1} \phi_{i}\left(x^{3}\right) e^{\rho i k x} d x+e^{\omega_{1} \rho k \pi} \int_{-y / 2}^{y} x^{i-1} \phi_{i}\left(x^{3}\right) e^{\rho i k x} d x \\
& +\left(-\omega_{3}\right)^{i+2} e^{\omega_{2} \rho i k \pi} \int_{-y / 2}^{-\omega_{2} y} x^{i-1} \phi_{i}\left(x^{3}\right) e^{\rho i k x} d x \\
& +\left(-\omega_{2}\right)^{i+2} e^{\omega_{3} \rho i k \pi} \int_{-y / 2}^{-\omega_{3} y} \lambda^{i-1} \phi_{i}\left(x^{3}\right) e^{\rho i k x} d x .
\end{aligned}
$$

These changes in the paths of integration, which are all taken as straight lines, can be made since the integrands are analytic functions of $x$ inside of $T_{a}$.

Now $\delta_{i}\left(\rho_{i k} \pi\right)=0$. The changes in the integral were made so that this fact could be used in showing that certain large portions of the integral cancel one another. Each of the three integrals whose lower limits are $-y / 2$ is sufficiently small to enable us to draw the desired conclusions about the coefficients. In fact, at no point of the paths of integration does the exponential factor take on an absolute value greater than its absolute value at the upper limit. Hence

$$
\left|\int_{-y / 2}^{-\omega_{i} y} x^{i-1} \phi_{i}\left(x^{3}\right) e^{\rho i k x} d x\right|<3 \pi M_{2}\left|e^{-\omega_{i} \rho_{i k} y}\right| / 2,
$$

where $M_{2}=\max \left|x^{i-1} \phi_{i}\left(x^{3}\right)\right|$ in and on the equilateral triangle centered at $x=0$ and having one vertex at the point $x=y$. Hence

* Ward, loc. cit., p. 741. 


$$
\left|\int_{0}^{\pi} x^{i-1} \phi_{i}\left(x^{3}\right) \delta_{3}\left[\rho_{i k}(\pi-x)\right] d x\right|<M \pi\left|e^{\omega_{3} \rho i k(x-y)}\right|,
$$

where $M$ has an obvious constant value.

Since

$$
\left|\delta_{j}\left(\rho_{i k} \pi\right)\right|>h\left|e^{\omega_{3} \rho_{i k} \pi}\right| \text { if } i \neq j^{*},
$$

where $h$ is independent of $k$, we have

$$
\left|a_{i k}\right|<M\left|e^{-\omega_{3} \rho i k y}\right| / h \text {. }
$$

If now $x$ is restricted to the interval $0 \leqq x \leqq \alpha<y$, we shall have

$$
\begin{aligned}
\left|a_{i k} \delta_{i}\left(\rho_{i k} x\right)\right| & <3 M\left|e^{\omega_{3} \rho_{i k}(x-y)}\right| / h \\
& \leqq 3 M\left|e^{\omega_{3} \rho_{i k}(\alpha-y)}\right| / h \quad(i=1,2,3) .
\end{aligned}
$$

This last expression, however, is the general term of a convergent series of positive constants. Consequently the series mentioned in the theorem converges to a function of $x$ analytic at every point interior to the smaller of $T_{a}$ and $T_{\pi}$. But it is known to converge to $x^{i-1} \phi_{i}\left(x^{3}\right)$ at every point interior to $T_{b}$. Hence, in view of the fact that $x^{i-1} \phi_{i}\left(x^{3}\right)$ is analytic at every interior point of $T_{a}$, the series converges uniformly to this function in and on the boundary of every closed region entirely within the smaller of $T_{a}$ and $T_{\pi}$.

If $|a|=\pi$, the triangle $T_{b}$ coincides with $T_{\pi}$, and Theorem I of part I of the 1927 paper tells us that $T_{x}$ is the triangle every closed interior region of which is a region of uniform convergence of the series.

We next consider uniform convergence of series (3) in the interval $0 \leqq x \leqq \pi$. For this it is evidently necessary that $\phi_{i}\left(\pi^{3}\right)=0$. The following theorem gives sufficient conditions for this convergence.

Theorem II. If $x^{i-1} \phi_{i}\left(x^{3}\right)$ is analytic at every point inside $T_{x}$, continuous together with its first and second derivatives on $T_{\pi}$, and zero at $\pi$, then the formal series (3) converges uniformly to $x^{i-1} \phi_{i}\left(x^{3}\right)$ inside and on $T_{x}$.

Since the method of proof is similar to that of Theorem I, we shall merely indicate the more important relations. Integrating by parts twice, we obtain

$$
\int_{0}^{\pi} x^{i-1} \phi_{i}\left(x^{3}\right) \delta_{3}\left[\rho_{i k}(\pi-x)\right] d x=\int_{0}^{\pi} \psi_{i}(x) \delta_{2}\left[\rho_{i k}(\pi-x)\right] d x / \rho_{i k}^{2},
$$

where $\psi_{i}(x)=\left(d^{2} / d x^{2}\right)\left[x^{i-1} \phi_{i}\left(x^{3}\right)\right]$ is analytic at every interior point of $T_{\pi}$ and continuous on $T_{\pi}$. Also $\psi_{i}\left(-\omega_{3} x\right)=\left(-\omega_{3}\right) \psi_{i}(x)$ and $\psi_{i}\left(-\omega_{2} x\right)$ $=\left(-\omega_{2}\right)^{i} \psi_{i}(x)$. Hence

* Ward, loc. cit., p. 719 (2). 


$$
\begin{gathered}
\int_{0}^{\pi} x^{i-1} \phi_{i}\left(x^{3}\right) \delta_{3}\left[\rho_{i k}(\pi-x)\right] d x \\
=\frac{1}{\rho_{i k}^{2}}\left[\delta_{i}\left(\rho_{i k} \pi\right) \int_{0}^{-\pi / 2} \psi_{i}(x) e^{\rho_{i k} x} d x+e^{\omega_{1} \rho i k \pi} \int_{-\pi / 2}^{\pi} \psi_{i}(x) e^{\rho_{i k} x} d x\right. \\
\left.+\left(-\omega_{3}\right)^{i+2} e^{\omega_{2} \rho i k \pi} \int_{-\pi / 2}^{-\omega_{2} \pi} \psi_{i}(x) e^{\rho_{i k} x} d x+\left(-\omega_{2}\right)^{i+2} e^{\omega_{3} \rho i k \pi} \int_{-\pi / 2}^{-\omega_{3} \pi} \psi_{i}(x) e^{\rho_{i k} x} d x\right] .
\end{gathered}
$$

Consequently

$$
\left|\int_{0}^{\pi} x^{i-1} \phi_{i}\left(x^{3}\right) \delta_{3}\left[\rho_{i k}(\pi-x)\right] d x\right|<M \pi / \rho_{i k}^{2},\left|a_{i k}\right|<M\left|e^{-\omega_{8} \rho i k \pi}\right| /\left(h \rho_{i k}^{2}\right),
$$

and $\left|a_{i k} \delta_{i}\left(\rho_{i k} x\right)\right|<3 M /\left(h \rho_{i k}^{2}\right)$ if $x$ is within or on $T_{\pi}$. Since $1 / \rho_{i k}^{2}$ is the general term of a convergent series of positive constants, this completes the proof.

Finally, we note that necessary conditions for the triangle of convergence to contain $\pi$ as an interior point are that $\left(d^{3 n} / d x^{3 n}\right)\left[x^{i-1} \phi_{i}\left(x^{3}\right)\right]$ vanish at $x=\pi$ for $n=0,1,2, \cdots$. This fact and the above theorems enable us to state in most cases exactly the triangle of uniform convergence of the series (1) associated with a given analytic function. They fail to give us this information in case the parts $x^{i-1} \phi_{i}\left(x^{3}\right)$ of $f(x)$ have all their singularities at exterior points of $T_{\pi}$ and at the same time $\left(d^{3 n} / d x^{3 n}\right)\left[x^{i-1} \phi_{i}\left(x^{3}\right)\right]$ vanish at $x=\pi$ for $n=0,1,2, \cdots$, and $i=1,2,3$; but we can say in such a case that the region of uniform convergence contains $T_{\boldsymbol{x}}$.

4. Differentiation and integration of formal series. Inasmuch as the formal series (3) converge uniformly and their terms are analytic functions, differentiation term by term at any interior point of the region of uniform convergence is permissible, and the derived series will converge uniformly to the derived function. In this section we enquire whether if the series is differentiated term by term a number of times which is a multiple of three, the derived series will be the formal series of the derived function. It is clear in the case of series (4) that the third derived series are not the formal series of the third derived functions.

THEOREM III. If $x^{i-1} \phi_{i}\left(x^{3}\right)$ is analytic in some closed region of which $x=0$ is an interior point, and has on the interval $0 \leqq x \leqq \pi$ a continuous fifth derivative, then a necessary and suffcient condition that the third derived series for this function be the formal series for the third derivative of the function is $\phi_{i}\left(\pi^{3}\right)=0$.

The series for the given function being $\sum_{k=1}^{\infty} a_{i k} u_{i k}(x)$, the third derived 
series is $-\sum_{k=1}^{\infty} a_{i k} \rho_{i k}^{3} u_{i k}(x)$. Let us denote by $b_{i k}$ the $k$ th coefficient in the formal series for the third derivative of the given function. Then

$$
\pi b_{i k} \delta_{i-1}\left(\rho_{i k} \pi\right)=\int_{0}^{\pi} \frac{d^{3}}{d x^{3}}\left[x^{i-1} \phi_{i}\left(x^{3}\right)\right] \delta_{3}\left[\rho_{i k}(\pi-x)\right] d x .
$$

The requirement that the given function have a continuous fifth derivative on the interval $(0, \pi)$ is made so that we may be sure that the formal series for the third derivative of this function converges to that third derivative.

Integrating by parts three times and making use of the formula for $a_{i k}$ gives

$$
\pi b_{i k} \delta_{i-1}\left(\rho_{i k} \pi\right)=3 \rho_{i k}^{2} \pi^{i-1} \phi_{i}\left(\pi^{3}\right)-\pi \rho_{i k}^{2} a_{i k} \delta_{i-1}\left(\rho_{i k} \pi\right),
$$

from which the truth of the theorem is clear.

Since the formal series (3) converge uniformly in some region surrounding $x=0$, term-by-term integration along a simple curve lying within the region is admissible. We enquire whether, if the three-fold integral of $u_{i k}(x)$ is taken as $-u_{i k}(x) / \rho_{i k}^{3}$, the thrice integrated series will be the formal series of any function, and in case it is, of what function.

On integrating (3) we get

$$
\int_{0}^{x} t^{i-1} \phi_{i}\left(t^{3}\right) d t=-\sum_{k=1}^{\infty} a_{i k}\left[\delta_{i+1}\left(\rho_{i k} x\right)-\delta_{i+1}(0)\right] / \rho_{i k}
$$

or

$$
\int_{0}^{x} t^{i-1} \phi_{i}\left(t^{3}\right) d t+C_{1 i}=-\sum_{k=1}^{\infty} a_{i k} \delta_{i+1}\left(\rho_{i k} x\right) / \rho_{i k},
$$

where $C_{1 i}=-\delta_{i+1}(0) \sum_{k=1}^{\infty} a_{i k} / \rho_{i k}$. Two more integrations yield

$$
\int_{0}^{x} \int_{0}^{s} \int_{0}^{r} t^{i-1} \phi_{i}\left(t^{3}\right) d t d r d s+C_{1 i} x^{2} / 2+C_{2 i} x+C_{3 i}=-\sum_{k=1}^{\infty} a_{i k} u_{i k}(x) / \rho_{i k}^{2},
$$

where $C_{2 i}=\delta_{i+2}(0) \sum_{k=1}^{\infty} a_{i k} / \rho_{i k}^{2}$ and $C_{3 i}=-\delta_{i}(0) \sum_{k=1}^{\infty} a_{i k} / \rho_{i k}^{3}$.

Now call

$$
\phi(x)=\int_{0}^{x} \int_{0}^{s} \int_{0}^{r} t^{i-1} \phi_{i}\left(t^{3}\right) d t d r d s
$$

and expand $\phi(x)+C_{1 i} x^{2} / 2+C_{2 i} x+C_{3 i}$ in formal series. Since $C_{11}=C_{12}=C_{21}$ $=C_{23}=C_{32}=C_{33}=0$, the formal expansion is of the form

$$
\phi(x)+C_{1 i} x^{2} / 2+C_{2 i} x+C_{3 i}=\sum_{k=1}^{\infty} b_{i k} u_{i k}(x),
$$

where 


$$
\pi b_{i k} \delta_{i-1}\left(\rho_{i k} \pi\right)=\int_{0}^{\pi}\left[\phi(x)+C_{1 i} x^{2} / 2+C_{2 i} x+C_{3 i}\right] \delta_{3}\left[\rho_{i k}(\pi-x)\right] d x,
$$

and this series converges uniformly to the function which gave rise to it. Three integrations by parts give us

$$
\text { (7) } \pi b_{i k} \delta_{i-1}\left(\rho_{i k} \pi\right)=3\left[\phi(\pi)+C_{1 i} \pi^{2} / 2+C_{2 i} \pi+C_{3 i}\right] / \rho_{i k}-\pi a_{i k} \delta_{i-1}\left(\rho_{i k} \pi\right) / \rho_{i k}^{3} .
$$

We shall now evaluate those $C$ 's which are not given above as being zero. Placing $x=0$ and $i=1$ in (6) gives $C_{31}=3 \sum_{k=1}^{\infty} b_{i k}$. But when $i=1$, (7) is

$$
\pi b_{1 k} \delta_{3}\left(\rho_{1 k} \pi\right)=3\left[\phi(\pi)+C_{31}\right] / \rho_{1 k}-\pi a_{1 k} \delta_{3}\left(\rho_{1 k} \pi\right) / \stackrel{3}{\rho_{1 k}} .
$$

Hence, eliminating $b_{1 k}$,

$$
\begin{aligned}
C_{31} & =3 \sum_{k=1}^{\infty}\left[\frac{3\left\{\phi(\pi)+C_{31}\right\}}{\pi \rho_{1 k} \delta_{3}\left(\rho_{1 k} \pi\right)}-\frac{a_{1 k}}{\rho_{1 k}^{3}}\right] \\
& =\frac{9}{\pi} \sum_{k=1}^{\infty} \frac{\phi(\pi)+C_{31}}{\rho_{1 k} \delta_{3}\left(\rho_{1 k} \pi\right)}+C_{31} .
\end{aligned}
$$

Consequently

$$
\left[\phi(\pi)+C_{81}\right] \sum_{k=1}^{\infty} 1 /\left[\rho_{1 k} \delta_{3}\left(\rho_{1 k} \pi\right)\right]=0 .
$$

Now the series $(3 / \pi) \sum_{k=1}^{\infty} \delta_{1}\left(\rho_{1 k} x\right) /\left[\rho_{1 k} \delta_{3}\left(\rho_{1 k} \pi\right)\right]$ converges uniformly to unity in every closed region within $T_{\boldsymbol{r}}$. Hence, placing $x=0$, we have $\pi / 9=\sum_{k=1}^{\infty} 1 /\left[\rho_{1 k} \delta_{3}\left(\rho_{1 k} \pi\right)\right]$, and therefore $C_{31}=-\phi(\pi)$.

Similarly, differentiating (6) with $i=2$ once and placing $x=0$ yields $C_{22}=-\phi(\pi) / \pi$, while differentiating twice with $i=3$ and placing $x=0$ yields $C_{13}=-2 \phi(\pi) / \pi^{2}$. Hence, placing these results in (7), we have $b_{i k}=-a_{i k} / \rho_{i k}^{\mathbf{3}}$. This we sum up in

ThEOREM IV. If the formal series for $x^{i-1} \phi_{i}\left(x^{3}\right)$ be integrated term by term three times with respect to $x$, the resulting series is the formal series for

$$
\phi(x)+C_{1 i} x^{2} / 2+C_{2 i} x+C_{3 i},
$$

where

$$
\begin{gathered}
\phi(x)=\int_{0}^{x} \int_{0}^{s} \int_{0}^{r} t^{i-1} \phi_{i}\left(t^{3}\right) d t d r d s, C_{11}=C_{12}=C_{21}=C_{23}=C_{32}=C_{33}=0, \\
C_{13}=-2 \phi(\pi) / \pi^{2}, \quad C_{22}=-\phi(\pi) / \pi, \quad C_{31}=-\phi(\pi) .
\end{gathered}
$$

5. Relations involving the coefficients. This section is concerned with 
relations analogous to the Hurwitz theorem on the Fourier coefficients.* If the series $\sum_{k=1}^{\infty} a_{3 k} u_{3 k}(x)$ converges uniformly to $f(x)$ in $0 \leqq x \leqq \pi$, multiplying by $f(\pi-x)$ and integrating from 0 to $\pi$ yields

$$
\pi \sum_{k=1}^{\infty} a_{3 k}^{2} \delta_{2}\left(\rho_{3 k} \pi\right)=\int_{0}^{\pi} f(x) f(\pi-x) d x .
$$

The series on the left hand side of this equation, however, will converge even if the formal series for $f(x)$ converges uniformly in an interval extending from 0 to slightly beyond $\pi / 2$, and thus arises the question as to whether its sum is the same as when the interval of uniform convergence extends to $\pi$. This and similar questions are answered in the following theorem.

ThEOREM V. If the formal series $\sum_{k=1}^{\infty} a_{i k} \delta_{i}\left(\rho_{i k} x\right)$ converges uniformly to $f(x)$ in the interval $0 \leqq x \leqq \pi / 2+\delta$, where $\delta$ is an arbitrary positive constant $\dagger$ then
(a) $\quad \pi \sum_{k=1}^{\infty} a_{1 k}^{2} \delta_{3}\left(\rho_{1 k} \pi\right) / \rho_{1 k}^{2}=\int_{0}^{\pi} \int_{\pi}^{x} \int_{\pi}^{8} f(x) f(\pi-t) d t d s d x$ if $i=1$,
(b) $\quad \pi \sum_{k=1}^{\infty} a_{2 k}^{2} \delta_{1}\left(\rho_{2 k} \pi\right) / \rho_{2 k}=\int_{0}^{\pi} \int_{\pi}^{x} f(x) f(\pi-s) d s d x \quad$ if $i=2$,
(c) $\pi \sum_{k=1}^{\infty} a_{3 k}^{2} \delta_{2}\left(\rho_{3 k} \pi\right)=\int_{0}^{\pi} f(x) f(\pi-x) d x \quad$ if $i=3$.

The proofs of these are similar to one another, and for that reason we present the proof of (b) only. From our hypothesis it follows that $\sum_{k=1}^{\infty} a_{2 k} \delta_{2}\left[\rho_{2 k}(\pi-s)\right]$ converges uniformly to $f(\pi-s)$ in the interval $\pi / 2$ $-\delta \leqq s \leqq \pi$. Hence

$$
\begin{aligned}
\int_{\pi / 2}^{\pi} \int_{\pi}^{x} f(x) f(\pi-s) d s d x & =\int_{\pi / 2}^{\pi} f(x) \sum_{k=1}^{\infty} a_{2 k} \int_{\pi}^{x} \delta_{2}\left[\rho_{2 k}(\pi-s)\right] d s d x \\
& =\int_{\pi / 2}^{\pi}\left[f(x) \sum_{k=1}^{\infty} a_{2 k} \delta_{3}\left[\rho_{2 k}(x)\right] / \rho_{2 k}\right] d x \\
& =\sum_{k=1}^{\infty} a_{2 k} \int_{\pi / 2}^{\pi} f(x) \delta_{3}\left[\rho_{2 k}(\pi-x)\right] d x / \rho_{2 k} .
\end{aligned}
$$

But, making use of (2) with $i=2$, this becomes

$$
\begin{aligned}
\int_{\pi / 2}^{\pi} \int_{x}^{x} f(x) f(\pi-s) d s d x=\sum_{k=1}^{\infty} a_{2 k} & {\left[\pi a_{2 k} \delta_{1}\left(\rho_{2 k} \pi\right)\right.} \\
& \left.-\int_{0}^{\pi / 2} f(x) \delta_{3}\left[\rho_{2 k}(\pi-x)\right] d x\right] / \rho_{2 k}
\end{aligned}
$$

* Cf. Whittaker and Watson, Modern Analysis, second edition, $\$ 9.5$.

$\dagger \delta$ can be made zero if the term-by-term twice derived series converges uniformly to $f^{\prime \prime}(x)$ in $0 \leqq x \leqq \pi / 2$. 


$$
\begin{aligned}
= & \pi \sum_{k=1}^{\infty} a_{2 k}^{2} \delta_{1}\left(\rho_{2 k} \pi\right) / \rho_{2 k}+\phi^{2}(\pi / 2) \\
& +\sum_{k=1}^{\infty} a_{2 k} \int_{0}^{\pi / 2} \phi(x) \delta_{2}\left[\rho_{2 k}(\pi-x)\right] d x,
\end{aligned}
$$

where $\phi(x)=\int_{0}^{x} f(s) d s$. Also,

$$
\begin{aligned}
\int_{0}^{\pi / 2} \int_{\pi}^{x} f(x) f(\pi-s) d s d x \\
=\int_{0}^{\pi / 2} f(x)\left[\int_{\pi}^{\pi / 2} f(\pi-s) d s+\int_{\pi / 2}^{x} f(\pi-s) d s\right] d x \\
=-\phi^{2}(\pi / 2)+\sum_{k=1}^{\infty} a_{2 k} \int_{0}^{\pi / 2} \delta_{2}\left(\rho_{2 k} x\right) \int_{\pi / 2}^{x} f(\pi-s) d s d x \\
=-\phi^{2}(\pi / 2)+\sum_{k=1}^{\infty} a_{2 k} \int_{0}^{\pi / 2} \delta_{3}\left(\rho_{2 k} x\right) f(\pi-x) d x / \rho_{2 k} \\
=-\phi^{2}(\pi / 2)+\sum_{k=1}^{\infty} a_{2 k}\left[\pi a_{2 k} \delta_{1}\left(\rho_{2 k} \pi\right)-\int_{0}^{\pi / 2} f(x) \delta_{3}\left[\rho_{2 k}(\pi-x)\right] d x\right] / \rho_{2 k} \\
=\pi \sum_{k=1}^{\infty} a_{2 k}^{2} \delta_{1}\left(\rho_{2 k} \pi\right) / \rho_{2 k}-\phi^{2}(\pi / 2)-\sum_{k=1}^{\infty} a_{2 k} \int_{0}^{\pi / 2} f(x) \delta_{3}\left[\rho_{2 k}(\pi-x)\right] d x / \rho_{2 k} .
\end{aligned}
$$

Hence

$$
\begin{aligned}
\int_{0}^{\pi} \int_{\pi}^{x} f(x) f(\pi-s) d s d x & =2 \pi \sum_{k=1}^{\infty} a_{2 k}^{2} \delta_{1}\left(\rho_{2 k} \pi\right) / \rho_{2 k} \\
& +\sum_{k=1}^{\infty} a_{2 k} \int_{0}^{\pi / 2} \phi(x) \delta_{2}\left[\rho_{2 k}(\pi-x)\right] d x \\
& -\sum_{k=1}^{\infty} a_{2 k} \int_{0}^{\pi / 2} f(x) \delta_{3}\left[\rho_{2 k}(\pi-x)\right] d x / \rho_{2 k} .
\end{aligned}
$$

We are now in a position to insert the series developments for $f(x)$ and $\phi(x)$ since these converge uniformly in the interval over which we wish to integrate.

We have

$$
\sum_{k=1}^{\infty} a_{2 k} \int_{0}^{\pi / 2} \phi(x) \delta_{2}\left[\rho_{2 k}(\pi-x)\right] d x=-\sum_{k=1}^{\infty} a_{2 k} \sum_{l=1}^{\infty} a_{2 l} c_{l k} / \rho_{2 l},
$$

where 


$$
c_{l k}=\int_{0}^{\pi / 2} \delta_{3}\left(\rho_{2 l} x\right) \delta_{2}\left[\rho_{2 k}(\pi-x)\right] d x
$$

and

$$
\sum_{k=1}^{\infty} a_{2 k} \int_{0}^{\pi / 2} f(x) \delta_{3}\left[\rho_{2 k}(\pi-x)\right] d x / \rho_{2 k}=\sum_{k=1}^{\infty} a_{2 k} \sum_{l=1}^{\infty} a_{2 l} d_{l k} / \rho_{2 k},
$$

where

$$
d_{l k}=\int_{0}^{\pi / 2} \delta_{2}\left(\rho_{2 l} x\right) \delta_{3}\left[\rho_{2 k}(\pi-x)\right] d x
$$

(9) Consider now the double series $\sum_{k, l=1}^{\infty} a_{2 k} a_{2 l} c_{l k} / \rho_{2 l}$. Integration gives

$$
c_{l k}=\left[\rho_{2 k}^{2} \delta_{3}\left(\rho_{2 l} \pi / 2\right) \delta_{3}\left(\rho_{2 k} \pi / 2\right)+\rho_{2 k} \rho_{2 l} \delta_{2}\left(\rho_{2 l} \pi / 2\right) \delta_{1}\left(\rho_{2 k} \pi / 2\right)\right.
$$

$$
\left.+\underset{\rho_{2 l} \delta_{1}}{2}\left(\rho_{2 l} \pi / 2\right) \delta_{2}\left(\rho_{2 k} \pi / 2\right)\right] /\left(\rho_{2 k}^{8}-\rho_{2 l}^{3}\right) \text { if } l \neq k,
$$

and $c_{k k}=\pi \delta_{1}\left(\rho_{2 k} \pi\right) / 2-\delta_{3}^{2}\left(\rho_{2 k} \pi / 2\right) / \rho_{2 k}$. Now

$$
\left|\delta_{j}\left(\rho_{2 l} \pi / 2\right)\right|<3 e^{\rho_{2 l} \pi / 4} .
$$

Hence

$$
\begin{aligned}
\left|c_{l k}\right| & <9 e^{\left(\rho_{2 l}+\rho_{2 k}\right) \pi / 4} \frac{\rho_{2 k}^{2}+\rho_{2 k} \rho_{2 l}+\rho_{2 l}^{2}}{\left|\rho_{2 k}^{3}-\rho_{2 l}^{3}\right|} \\
& =9 e^{\left(\rho_{2 l}+\rho_{2 k}\right) \pi / 4} /\left|\rho_{2 k}-\rho_{2 l}\right| \text { if } l \neq k .
\end{aligned}
$$

Also

$$
\left|c_{k k}\right|<h e^{\rho_{2 k} \pi / 2} \text {, }
$$

where $h$ is independent of $k$. Hence

$$
\left|c_{l k}\right|<g e^{\left(\rho_{2 l}+\rho_{2 k}\right) \pi / 4}
$$

where $k$ and $l$ are not necessarily different and $g$ is independent of $k$ and $l$.

Furthermore,

$$
\left|a_{2 k}\right|<m e^{-p_{2 k} x_{1} / 2}
$$

where $\pi / 2<x_{1}<\pi / 2+\delta$ and $m$ and $x_{1}$ are independent of $k .{ }^{*}$ Hence

$$
\left|a_{2 k} a_{2 l} c_{l k} / \rho_{2 l}\right|<m^{2} g e^{\left(\rho_{2 l}+\rho_{2 k}\right)\left(x / 4-x_{2} / 2\right)} \text {. }
$$

This is recognized as the general term of a convergent double series. Hence series (9) converges absolutely.

\footnotetext{
* Ward, loc. cit., Lemma, p. 720.
} 
In the same way the double series $\sum_{k, l=1}^{\infty} a_{2 k} a_{2 l} d_{l k} / \rho_{2 k}$ can be shown to converge absolutely. We are justified, therefore, in summing series (9) first over $k$ and then over $l$ and adding to the series in the $d$ 's. This results in

$$
\sum_{k, l=1}^{\infty} a_{2 k} a_{2 l}\left(c_{k l}+d_{l k}\right) / \rho_{2 k} .
$$

Now $c_{k l}+d_{l k}=0$ or $\pi \delta_{1}\left(\rho_{2 k} \pi\right)$ according as $l$ is not or is equal to $k$, as is easily shown by noting that

$$
c_{k l}+d_{l k}=\int_{0}^{\pi} \delta_{3}\left(\rho_{2 l} x\right) \delta_{2}\left[\rho_{2 k}(\pi-x)\right] d x
$$

and performing the indicated integration.

Hence the double series (10) reduces to the simple series $\pi \sum_{k=1}^{\infty} a_{2 k}^{2} \delta_{1}\left(\rho_{2 k} \pi\right)$ $/ \rho_{2 b}$, and this with (8) proves part (b).

We note that if the second derived series of the series of the theorem converges uniformly to $f^{\prime \prime}(x)$ in $0 \leqq x \leqq \pi$, a simpler relation can be derived. In fact, multiplying $f^{\prime \prime}(\pi-x)=\sum_{k=1}^{\infty} a_{2 k} \rho_{2 k}^{2} \delta_{3}\left[\rho_{2 k}(\pi-x)\right]$ by $f(x)$ and integrating from 0 to $\pi$ gives

$$
\int_{0}^{\pi} f(x) f^{\prime \prime}(\pi-x) d x=\pi \sum_{k=1}^{\infty} a_{2 k \rho_{2 k} \delta_{1}}^{2}\left(\rho_{2 k} \pi\right) .
$$

However, this relation is not in general true if the interval of uniform convergence does not extend as far as $\pi$.

6. Determination of formal coefficients. The determination of the formal coefficients by rendering stationary certain integrals is of special interest in the expansion problems here considered because the integrals are similar to those of the preceding section.

ThEOREM VI. Let $f(x)$ be a function capable of development into series (3) for a definite value of $i$. If $S_{i n}(x)=\sum_{k=1}^{n} b_{i k} \delta_{i}\left(\rho_{i k} x\right)$ and $R_{i n}(x)=f(x)$ $-S_{\text {in }}(x)$, where $n$ is regarded as a constant and the b's as independent variables, then the following integrals have stationary values when the b's are equal to the formal coefficients:

$$
\begin{array}{ll}
\int_{0}^{\pi} \int_{\pi}^{x} \int_{\pi} R_{n}(x) R_{n}(\pi-t) d t d s d x & \text { in case } i=1, \\
\int_{0}^{\pi} \int_{\pi}^{x} R_{n}(x) R_{n}(\pi-s) d s d x & \text { in case } i=2, \\
\int_{0}^{\pi} R_{n}(x) R_{n}(\pi-x) d x & \text { in case } i=3 .
\end{array}
$$


Again we shall give the proof of part (b) only, the proofs of the other parts being very similar to it. We have

$$
\begin{aligned}
\int_{\pi}^{x} R_{n}(\pi-s) d s & =\int_{\pi}^{x} f(\pi-s) d s-\int_{\pi}^{x} \sum_{k=1}^{n} b_{2 k} \delta_{2}\left[\rho_{2 k}(\pi-s)\right] d s \\
& =\int_{\pi}^{x} f(\pi-s) d s-T_{n}(x),
\end{aligned}
$$

where $T_{n}(x)=\sum_{k=1}^{n} b_{2 k} \delta_{3}\left[\rho_{2 k}(\pi-x)\right] / \rho_{2 k}$. Hence, denoting by $I_{n}$ the integral to be rendered stationary,

$$
\begin{aligned}
I_{n}= & \int_{0}^{\pi} \int_{\pi}^{x} f(x) f(\pi-s) d s d x-\int_{0}^{\pi} f(x) T_{n}(x) d x \\
& -\int_{0}^{\pi} \int_{\pi}^{x} S_{n}(x) f(\pi-s) d s d x+\int_{0}^{\pi} S_{n}(x) T_{n}(x) d x,
\end{aligned}
$$

and

But

$$
\begin{aligned}
\frac{\partial I_{n}}{\partial b_{2 k}}= & -\int_{0}^{\pi} f(x) \frac{\partial T_{n}(x)}{\partial b_{2 k}} d x-\int_{0}^{\pi} \int_{\pi}^{x} f(\pi-s) \frac{\partial S_{n}(x)}{\partial b_{2 k}} d s d x \\
& +\int_{0}^{\pi} S_{n}(x) \frac{\partial T_{n}(x)}{\partial b_{2 k}} d x+\int_{0}^{\pi} \frac{\partial S_{n}(x)}{\partial b_{2 k}} T_{n}(x) d x .
\end{aligned}
$$

and

$$
\frac{\partial S_{n}(x)}{\partial b_{2 k}}=\delta_{2}\left(\rho_{2 k} x\right)
$$

$$
\frac{\partial T_{n}(x)}{\partial b_{2 k}}=\delta_{3}\left[\rho_{2 k}(\pi-x)\right] / \rho_{2 k} .
$$

Putting in these values and remembering (11) this becomes

$$
\begin{aligned}
\frac{\partial I_{n}}{\partial b_{2 k}}= & -\int_{0}^{\pi} f(x) \delta_{3}\left[\rho_{2 k}(\pi-x)\right] d x / \rho_{2 k} \\
& -\int_{0}^{\pi} \int_{\pi}^{x} f(\pi-s) \delta_{2}\left(\rho_{2 k} x\right) d s d x+2 \pi b_{2 k} \delta_{1}\left(\rho_{2 k} \pi\right) / \rho_{2 k} .
\end{aligned}
$$

But, by integration by parts,

$$
\int_{0}^{\pi} \int_{\pi}^{x} f(\pi-s) \delta_{2}\left(\rho_{2 k} x\right) d s d x=\int_{0}^{\pi} f(\pi-x) \delta_{3}\left(\rho_{2 k} x\right) d x / \rho_{2 k} .
$$


Changing the variable of integration to $x^{\prime}$, where $x^{\prime}=\pi-x$, and substituting in (12) results in

$$
\frac{\partial I_{n}}{\partial b_{2 k}}=2\left[-\int_{0}^{\pi} f(x) \delta_{3}\left[\rho_{2 k}(\pi-x)\right] d x+\pi b_{2 k} \delta_{1}\left(\rho_{2 k} \pi\right)\right] / \rho_{2 k},
$$

which, on comparison with (2), completes the proof.

UNIVERSITY OF IOWA, Iowa City, Iowa 